\title{
Über die synthetische Darstellung des Iretols und einiger verwandter Phentetrolderivate von
}

\section{Emil Kohner.}

(Aus dem I. chemischen Laboratorium der k. k. Universität in Wien.)

(Vorgelegt in der Sitzung am 3. November 1899.)

Der Ersatz von Amidogruppen durch Hydroxylgruppen bei der Einwirkung von Wasser bei der Siedetemperatur, unter Atmosphärendruck und bei neutraler Reaction hat sich bis jetzt nur im symmetrischen Triamidobenzol ${ }^{1}$ und dessen Homologen ${ }^{2}$ in glatter Weise bewerkstelligen lassen.

Auf diesem Wege konnte man das Phloroglucin und einige Homologe desselben in günstiger Ausbeute und grosser Reinheit gewinnen.

Von anderen Triamidoverbindungen ist bezüglich des Verhaltens gegen Wasser nur das 1,2,4,6-Triamidophenol einer eingehenden Untersuchung gewürdigt worden $;^{3}$ dieselbe ergab, dass nur zwei Amidogruppen bei $100^{\circ}$ und gewöhnlichem Druck in Hydroxylgruppen übergehen, die dritte Amidogruppe aber erst durch Erhitzen des auf diese Weise gewonnenen Trioxyanilins mit Salzsäure unter erhöhtem Druck. Die Ausbeute des nach diesem ziemlich umständlichen Verfahren gewonnenen Tetraoxybenzols lässt sich mit den günstigen Ausbeuten an den Phloroglucinen kaum vergleichen.

Das verschiedene Verhalten gegen Wasser, welches das Triamidophenol im Vergleiche zum Triamidobenzol zeigt, hat

1 Monatshefte fïr Chemie, 18,755 .

2 Ibid., 19, 223, 236, 249.

3 Monatshefte für Chemie, 16, 248. 
mich veranlasst, die Gruppe der Triamidophenols in ihrem Verhalten zum Wasser zu studiren.

Zunächst konnte ich mich durch Versuche überzeugen, dass auch bei der Einwirkung von Wasser auf das Triamidophenol selbst, wenn auch in überaus geringer Menge, ein von Stickstoff freier Körper sich bildet, der sich mit Äther ausschütteln lässt und den Schmelzpunkt $164^{\circ}$ zeigt. Mit Rücksicht auf die schlechte Ausbeute dieses offenbar mit dem von Öttinger dargestellten ${ }^{1}$ Phentetrol identischen Productes habe ich auf die zur Reinigung und exacten Identificirung nothwendige Darstellung grösserer Mengen dieses Körpers verzichtet.

Ein hinderndes Moment bei der Hydrolysirung des Triamidophenols ist offenbar die Parastellung einer Amidogruppe zur Hydroxylgruppe, welche stets die Möglichkeit einer Bildung chinonartiger Nebenproducte sehr zersetzlicher Natur in sich schliesst.

Ist aber der Eintritt derartiger Nebenreactionen durch Einführung von Alkyl an die Stelle von Hydroxylwasserstoff erschwert, so war zu erwarten, dass diese Triamidophenoläther in ihrem Verhalten gegen Wasser den Homologen des Triamidobenzols ähnlich sein würden.

Der Versuch, in das Triamidophenol eine Alkylgruppe einzuführen, erschien mir in Folge der grossen Zersetzlichkeit des freien Triamidophenols aussichtslos, und so blieb nur der indirecte Weg zur Darstellung derartiger Verbindungen aus den leicht zugänglichen Äthern der Pikrinsäure übrig.

Das grösste Interesse nahm der Methyläther des Triamidophenols in Anspruch, weil dessen Hydrolysirung im Falle des Gelingens zu dem von de Laire und Tiemann beschriebenen, als Spaltungsproduct des Irigenins gewonnenen Iretol ${ }^{2}$ führen musste. Zugleich läge in dieser Synthese des Iretols ein zwingender Constitutionsbeweis für diesen Körper, dessen Structurformel sich bisher hauptsächlich auf seinen Übergang in Phloroglucin bei der Einwirkung von Natriumamalgam stützte.

1 Monatshefte für Chemie, 16, 248.

2 Berl. Ber., 26, 2015. 
Die Darstellung des Pikrinsäuremethyläthers habe ich nach den Angaben von Cahours ${ }^{1}$ durch Erhitzen von Anissäure mit einem Gemische von rauchender Schwefelsäure und rauchender. Salpetersäure auf circa $95^{\circ}$ vorgenommen; nach zwei- bis dreimaligem Umkrystallisiren aus absolutem Alkohol zeigte derselbe den richtigen Schmelzpunkt und erwies sich als analysenrein.

\section{Reduction des Pikrinsäuremethyläthers durch Zinn und Salzsäure.}

Um einer partiellen Abspaltung der Methylgruppe in Form von Chlormethyl vorzubeugen, schien es rathsam, die Reduction mit verdünnter Salzsäure vorzunehmen, jede äussere Wärmezufuhr thunlichst $z \mathrm{u}$ vermeiden und das Reactionsgemisch etrforderlichen Falles abzukühlen.

Spätere Versuche haben mich überzeugt, dass diese Vorsicht sehr am Platze war; denn bei Anwendung concentrirter Salzsäure verläuft die Reaction stürmisch, und das Reductionsproduct erweist sich als ein. Gemenge zweier Körper, deren einer methoxylfrei ist.

Folgendes Verfahren lässt die günstigsten Resultate erzielen: $15 \mathrm{~g}$ fein zerriebenen Pikrinsäuremethyläthers und $60 \mathrm{~g}$ $Z$ inn wurden in einem Kolben mit einer Mischung von $150 \mathrm{~cm}^{3}$ Salzsäure vom specifischen Gewichte 1.18 und $50 \mathrm{~cm}^{3}$ Wasser übergossen. $100 \mathrm{~cm}^{3}$ Wasser bält man in Reserve, um dieselben im Verlaufe der Reaction allmälig zuzusetzen.

Man überlässt das Reactionsgemisch zunächst sich selbst; unter mässig lebhafter Wasserstoffentwicklung erwärmt sich dasselbe allmälig, und der geschmolzene Nitrokörper schwimmt schliesslich als Ölschichte obenauf. Reduction und Lösung desselben, welche ziemlich langsam (beiläufig in einer halben Stunde) vor sich geht, kann man durch öfteres Umschwenken des Kolbens befördern, keinesfalls aber soll die Temperatur über $75^{\circ}$ steigen; die'letzten Öltropfen, welche der Lösung meistens hartnäckig widerstehen, kann man durch vorsichtiges Erwärmen am Wasserbade zum Verschwinden bringen.

1 Ann. Chem. und Pharm., 69, 238. 
Sobald Alles, bis auf unlösliche Verunreinigungen des Zinns, in Lösung gegangen ist, lässt man die bräunlichgelb gefärbte Flüssigkeit ein wenig abkühlen, filtrirt über Glaswolle und destillirt die Lösung im partiellen Kohlensäurevacuum bei $50-60^{\circ}$ ab. Der Rückstand wird in Wasser gelöst, mit Schwefelwasserstoff entzinnt und die vom Schwefelzinn getrennte Flüssigkeit wie früher durch Destillation eingeengt. Bei genügender Concentration scheidet sich das salzsaure Salz des Amidoproductes beim Erkalten in farblosen Krystallkrusten aus. Man saugt diese Ausscheidung ab und kann durch Einengen der Lauge, welche sich an der Luft rasch röthlich färbt, weitere Mengen des Körpers erhalten. Dieser färbt sich schon beim Abfiltriren blassviolett; man wäscht mit etwas concentrirter Salzsäure nach und reinigt das so erhaltene Rohproduct durch Umkrystallisiren aus circa vierprocentiger Salzsäure.

Löst man den Körper in der Siedehitze in der eben hinreichenden Menge von Salzsäure der genannten Concentration auf, so erstarrt die niemals ganz farblos zu erhaltende Lösung beim Erkalten allmälig zu einem dicken Brei anscheinend farbloser, zu baum- oder farnkrautartigen Complexen vereinigter Nädelchen. Dieselben nehmen aber schon beim Abfiltriren wieder eine blassviolette Färbung an. Bei längerem Verweilen an feuchter Luft verwandeln sich die Krystalle in gelbbraune schmierige Massen, dagegen lassen sie sich in trockenen, gut verschlossenen Gefässen' wochenlang unverändert aufbewahren. Die wässerige Lösung der Verbindung, welche blass röthlich gefärbt ist, gibt mit verdünnter Eisenchloridlösung keine charakteristische Färbung, sondern dieselbe färbt sich, wie dies bei vielen zersetzlichen Körpern zu beobachten ist, allmälig gelbbraun. Alkalien und Ammoniak rufen ähnliche Färbungen hervor. Beim Erhitzen der trockenen Verbindung auf circa $120^{\circ}$ tritt Gelbfärbung, bei noch höherer Temperatur Zersetzung zu braunen, theerartigen, sehr schwer verbrennbaren Massen ein.

Die Analyse der im Vacuum bis zur Gewichtsconstanz getrockneten Substanz ergab die folgenden Werthe:

I. $0 \cdot 2304 \mathrm{~g}$ Substanz gaben $0 \cdot 3125 g$. Kohlensäure und $0 \cdot 1084 \mathrm{~g}$ Wasser.

II. $0.2242 \mathrm{~g}$ Substanz gaben bei $19^{\circ} \mathrm{C}$. und $753.5 \mathrm{~mm}$ Druck $23.3 \mathrm{~cm}^{3}$ feuchten Stickstoff. 
III. $0 \cdot 2434 \mathrm{~g}$ Substanz gaben $0 \cdot 309 \mathrm{~g}$ Chlorsilber.

IV. $0 \cdot 2307 \mathrm{~g}$ Substanz gaben $0 \cdot 2385 \mathrm{~g}$ Jodsilber nach Z eisel.

In 100 Theilen:

$\begin{array}{lcccc} & \text { I } & \text { II } & \text { III } & \text { IV } \\ \mathrm{C} \ldots \ldots \ldots \ldots & 36 \cdot 99 & - & - & - \\ \mathrm{H} \ldots \ldots \ldots \ldots & 5 \cdot 22 & - & - & - \\ \mathrm{N} \ldots \ldots \ldots \ldots & - & 11 \cdot 84 & - & - \\ \mathrm{C} 1 \ldots \ldots \ldots & - & - & 31 \cdot 34 & - \\ \mathrm{OCH}_{3} \ldots \ldots & - & - & - & 13.68\end{array}$

Diese Zahlen stehen, wie nachfolgende Tabelle zeigt, mit den für das Chlorhydrat des Triamidophenolmethyläthers berechneten Zahlen nicht in Übereinstimmung, sondern führen zur Annahme der Formel $\mathrm{C}_{6} \mathrm{H}_{2}\left(\mathrm{NH}_{2}\right)_{2}(\mathrm{OH}) \mathrm{OCH}_{3} 2 \mathrm{HCl}$.

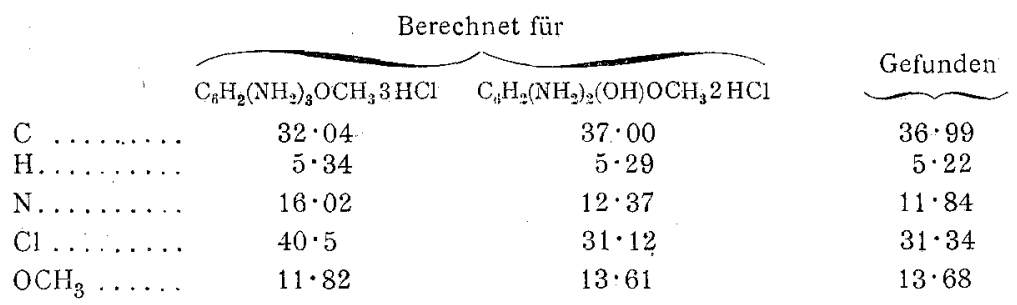

Es ergibt sich nun die Frage, ob der Ersatz der einen Amidogruppe durch Hydroxyl schon während der Reduction oder erst beim Eindampfen der zinnhaltigen, beziehungsweise der entzinnten Lösung vor sich geht. Folgende Thatsachen sprechen für erstere Annahme.

$20 \mathrm{~g}$ Nitrokörper wurden auf die oben beschriebene Weise reducirt und in die bei der Reduction erhaltene zinnchlorürhaltige Flüssigkeit gasförmiger Chlorwasserstoff eingeleitet. Es erfolgte die Ausscheidung eines farblosen krystallinischen Niederschlages von nicht unbeträchtlicher Quantität; ich will denselben mit $B$, die mit Salzsäure gesättigte Lösung mit $A$ bezeichnen. $B$ wurde filtrirt und getrocknet und mit Schwefelwasserstoff behandelt, ebenso wurde $A$ auf die gewohnte Weise entzinnt und aus beiden Partien, natürlich getrennt von einander, die in ihnen enthâltene Substanz möglichst vollkommen zur Abscheidung gebracht. Die Lösung $A$ lieferte 
circa $12 \mathrm{~g}$ des Chlorhydrates, das zu Folge der Analyse eine ganz geringe Verunreinigung von Chlorammonium enthielt. $B$ dagegen lieferte insgesammt $3 g$ feste Substanz; und zwar schieden sich zuerst Krystalle aus, die sich schon äusserlich durch ihre Farblosigkeit und ihre abweichende Krystallform von dem leicht $\mathrm{zu}$ erkennenden Chlorhydrat unterschieden; diese Ausscheidung bestand aus nahezu reinem Chlorammonium, ihre Menge betrug $2 g$; der Rest von beiläufig $1 \mathrm{~g}$ erwies sich als durch Chlorammonium stark verunreinigtes Chlorhydrat.

Das hier gefunderie Chlorammonium verdankt seine Entstehung offenbar dem Ersatz einer Amidogruppe durch die Hydroxylgruppe. Dieser Process spielt sich nach folgender Gleichung ab:

$$
\begin{aligned}
& \mathrm{C}_{6} \mathrm{H}_{2}\left(\mathrm{OCH}_{3}\right)\left(\mathrm{NH}_{2}\right)_{3} 3 \mathrm{HCl}+ \mathrm{H}_{2} \mathrm{O}= \\
& \mathrm{NH}_{4} \mathrm{Cl}+\mathrm{C}_{6} \mathrm{H}_{2}\left(\mathrm{OCH}_{3}\right) \mathrm{OH}\left(\mathrm{NH}_{2}\right)_{2} 2 \mathrm{HCl},
\end{aligned}
$$

und es entspricht daher der erhaltenen Menge von $13 \mathrm{~g}$ Chlorhydrat $2 \frac{1}{2} g$ Salmiak. Die Hauptmenge dieser theoretisch geforderten Quantität wurde in der Ausscheidung $B$ gefunden, während die Hauptmenge des entstandenen Reductionsproductes im Filtrat ' $A$ enthalten' war. Daraus ergibt sich mit Evidenz, dass die Gesammtmenge des entstandenen Chlorammoniums unmittelbar nach der Reduction bereits vorhanden sein musste; demzufolge kann der Ersatz der Amidogruppe durch Hydroxyl nicht etwa erst beim Eindampfen der entzinnten Lösungen erfolgt sein, sondern Reduction und Hydrolysirung fanden gleichzeitig oder unmittelbar nach einander statt.

Der Ersatz einer Amidogruppe durch Hydroxyl bei der Einwirkung von Zinn und Salzsäure auf Nitrokörper ist übrigens keine neue Thatsache; dieselbe wurde unter Anderem schon bei der Reduction des 1,2,4,6-Trinitroanilins ${ }^{1}$ (Pikramid) beobachtet. Dasselbe liefert nicht das zu erwartende 1,2,4,6Tetramidobenzol, sondern durch Versetzen der entzinnten Lösung mit concentrirter Salzsäure in der Kälte wird 1, 2, 4,6Triamidophenol gefällt

1. Ann. Chem. und Pharm., 215, 350. 
Einwirkung von Essigsäureanhydrid auf das Chlorhydrat des Diamidodioxybenzolmethyläthers.

$20 \mathrm{~g}$ salzsaures Salz, das nicht erst durch Umkrystallisiren gereinigt worden war, wurden mit der achtfachen Menge Essigsäureanhydrid übergossen und fünf Stunden im Wasserbad auf $100^{\circ}$ erhitzt. Hierauf wurde das überschüssige Essigsäureanhydrid im Vacuum abdestillirt. Es hinterblieb eine braune zähe Masse, die ich durch Digeriren mit absolutem Alkohol zum grössten Theil in Lösung brachte. Die alkoholische Lösung wurde im Wasserbad eingedampft und der Rückstand bei gelinder Wärme in Wasser gelöst; beim Eindampfen dieser Lösung, welche braun gefärbt war und nach Essigsäure roch, schieden sich alsbald glänzende Krystallkrusten aus, welche, abgesaugt und mit etwas kaltem Wasser gewaschen, eine gelbliche Färbung zeigten.

Das Product wurde nun bei gelinder Wärme in verdünntem Alkohol gelöst und mit Thierkohle behandelt; aus der heiss filtrirten Lösung schieden sich beim Erkalten farblose Nadeln aus, deren Menge beim Eindampfen der Lösung noch zunahm. Nach wiederholtem Umkrystallisiren aus Wasser zeigten dieselben den constanten Schmelzpunkt 194-196 ${ }^{\circ}$ (uncorr.).

Der Körper ist in kaltem Wasser schwer, in heissem Wasser dagegen ziemlich leicht löslich, ebenso in Alkohol, dagegen sehr schwer löslich in Benzol, Xylol und Äther.

Die Analyse der bei $100^{\circ}$ getrockneten Substanz ergab die folgenden Werthe:

I. $0.2502 \mathrm{~g}$ Substanz lieferte $0.5107 \mathrm{~g}$ Kohlensüure und $0.1317 \mathrm{~g}$ Wasser.

II. $0.2464 \mathrm{~g}$ Substanz lieferte $21.5 \mathrm{~cm}^{3}$ feuchten Stickstoff bei $748 \cdot 6 \mathrm{~mm}$ Druck und $17^{\circ} \mathrm{C}$.

III. $0.2475 \mathrm{~g}$ Substanz gaben $0.2147 \mathrm{~g}$ Jodsilber nach $\mathrm{Z}$ eisel.

In 100 Theilen:

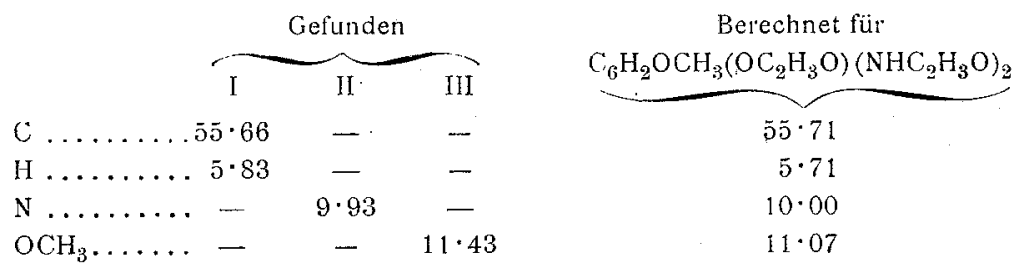


Aus diesen Zahlen ergibt sich, dass die Acetylirung des Diamidodioxybenzolmethylätherbichlorhydrates zur Bildung eines Triacetylderivates führt.

\section{Einwirkung von Wasser auf das Chlorhydrat des Dioxy- diamidobenzolmethyläthers.}

Da die nach dem Vorgange von Öttinger ${ }^{1}$ ausgeführte Hydrolyse ungünstige Ausbeuten ergab, wurde dieselbe in der Weise modificirt, dass zur Hintanhaltung von Oxydationsprocessen der Lösung etwas Zinnchlorür beigefügt wurde. Folgende Mengenverhältnisse ergaben die besten Resultate.

$25 \mathrm{~g}$ des durch die Reduction gewonnenen und vollständị von Salzsäure befreiten trockenen Rohproductes und $5 g 70$ procentiges Zinnchlorür wurden in $1 \frac{1}{2} l$ luftfreien Wassers gelöst und unter Durchleiten eines Kohlensäurestromes die Lösung während 24 Stunden unter Rückfluss zum Sieden erhitzt. Die rothgelb gefärbte und durch spärlich ausgeschiedene, braune Flocken etwas getrübte Flüssigkeit wurde sodann im Kohlensäurestrom erkalten gelassen, filtrirt und mit Schwefelwasserstoff entzinnt.

Die vom Schwefelzinn abfiltrirte Flüssigkeit, welche schwach gelb gefärbt war, wurde nun durch Vacuumdestillation auf circa $400 \mathrm{~cm}^{3}$ concentrirt und nach dem Erkalten wiederholt mit Äther ausgeschüttelt. Die ätherischen Auszüge wurden zunächst am Wasserbad eingeengt und sodann im Vacuum bei Zimmertemperatur vollständig abgedunstet; es hinterblieb ein gelbbraun gefärbter Syrup, der beim Reiben mit dem Glasstabe krystallinisch erstarrte.

Der Körper wurde in Wasser unter gelindem Erwärmen gelöst und mit einigen Tropfen einer concentrirten Bleizuckerlösung versetzt; die Lösung wurde von dem sich ausscheidenden gallertigen, braungelben Niederschlag abfiltrirt und im Filtrat das Blei mit Schwefelwasserstoff gefällt; die vom Schwefelblei abfiltrirte Flüssigkeit wurde im Vacuum bis zur Trockene abdestillirt, dann in der eben hinreichenden Menge Essigäther gelöst, filtrirt und mit dem $z$ wei- bis dreifachen Volumen Chloroform versetzt.

1 Monatshefte für Chemie, 16, 248. 
Nach kurzer Zeit schied sich die Verbindung in schönen Nädelchen aus, die beinahe farblos waren und nach mehrmaliger Wiederholung des oben beschriebenen Reinigungs. verfahrens den constanten Schmelzpunkt $186^{\circ}$ zeigten,

Die Analyse der bei $100^{\circ}$ getrockneten Substanz ergab die folgenden Werthe:

I. $0.2141 \mathrm{~g}$ Substanz gaben $0.4196 \mathrm{~g}$ Kohlensäure und $0.99 \mathrm{~g}$ Wasser.

II. $0 \cdot 2131 g$ Substanz gaben $0 \cdot 3196 g$ Jodsilber nach $Z$ e is el.

Auf 100 Theil

\begin{tabular}{|c|c|c|}
\hline \multicolumn{2}{|c|}{ Gefunden } & \multirow{2}{*}{$\begin{array}{r}\text { Berechnet für } \\
\mathrm{C}_{6} \mathrm{H}_{2} \mathrm{OCH} \mathrm{H}_{3}(\mathrm{OH})\end{array}$} \\
\hline $\bar{I}$ & $\mathrm{II}$ & \\
\hline$\ldots \ldots \ldots, 53 \cdot 43$ & - & $53 \cdot 84$ \\
\hline $\mathrm{H} \ldots \ldots, \ldots \ldots, 5 \cdot 14$ & - & $5 \cdot 13$ \\
\hline $\mathrm{OCH}_{3} \cdot \ldots . \quad-$ & $19 \cdot 83$ & $19 \cdot 98$ \\
\hline
\end{tabular}

Mithin stehen die gefundenen Zahlen mit den für den Phentetrolmonomethyläther vorausgesehenen in genügender Übereinstimmung.

Wie bereits ausgeführt ist, musste der aus dem Reductionsproduct des Pikrinsäuremethyläthers durch vollkommene Hydrolysé entstehende Körper mit dem von de Laire und Tiemann erhaltenen Iretol unter Voraussetzung der Richtigkeit der von diesen Autoren aufgestellten Structurformel identisch sein.

In der That haben' die genannten Forscher beim Iretol, welches sie einem ähnlichen Reinigungsverfahren unterworfer. haben, denselben Schmelzpunkt $\left(186^{\circ}\right)$ erhalten. Auch die anderen Eigenschaften beider Producte stimmen überein. Der von mir dargestellte Körper ist gleich dem Iretol in Wasser, Alkohol, Essigäther leicht, in trockenem Äther, Chloroform und Xylol sehr schwer löslich; er gibt die von de Laire und Tiemann beschriebene Eisenchlorid- und Fichtenspahnreaction, und es kann daher keinem $Z$ weifel unterliegen, dass er mit dem Iretol identisch ist.

Die Stellung der Hydroxylgruppen geht aus seinen genetischen Beżiehungen zum Methyläther der Pikrinsäure hervor, da sich das Iretol aus demselben durch zwei erfahrungsgemäss ohne Umlagerung sich vollziehende Reactionen gewinnen lässt. 
<smiles>CO[C@H]1C[N+](=O)[C@H]([N+](=O)[O-])C[C@H]1[N+](=O)[O-]</smiles>

I

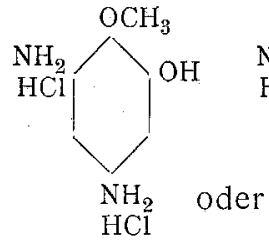

II

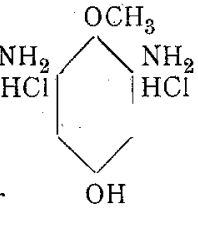

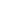<smiles>COC1CC(O)CCO1</smiles>

III

Die aus diesen Beziehungen sich ergebende Ortsbestimmung stimmt mit der von de Laire und Tieman $\mathrm{n}$ angegebenen überein.

Mit Rücksicht auf die vollkommen erwiesene Identität habe ich eine Darstellung von bereits bekannten Derivaten des Iretols, deren eine Anzahl von de Laire und Tiemann gewonnen wurden, nicht vorgenommen, sondern mich auf die Darstellung des bisher nicht beschriebenen Acetylproductes beschränkt.

\section{Einwirkung von Essigsäureanhydrid auf Iretol.}

$2 g$ ungereinigtes Iretol wurden mit der zehnfachen Menge Essigsäureanhydrid vier Stunden auf $100^{\circ}$ erhitzt, hierauf die Lösung auf ganz kurze Zeit zum Sieden erhitzt und nach dem Erkalten das überschüssige Essigsäureanhydrid im Vacuum abdestillirt. Da es nicht gelang, den zähen, braun gefärbten Rückstand zum Krystallisiren zu bringen, habe ich den Körper durch Destillation zu reinigen versucht; der grösste Theil ging unter $25 \mathrm{~mm}$ Druck bei $230^{\circ}$ über in Form eines dicken, gelblich gefärbten Syrups, der bei $-10^{\circ}$ fest wird, ohne zu krystallisiren und bei Zimmertemperatur sich wieder verflüssigt. Ich habe diesen Syrup einer nochmaligen Destillation unterworfen, wobei fast die ganze Menge bei dem constanten Siedepunkt $230^{\circ}$ unter $25 \mathrm{~mm}$ Druck überdestillirte. Das schwach gelblich gefärbte Destillat wurde in einem verkorkten Röhrchen 4-5 Tage bei Zimmertemperatur stehen gelassen; nach dieser Zeit begannen sich allmälig Krystallnadeln auszuscheidèn, und nach ungefähr 2 Tagen war die ganze Masse zu einem farblosen krystallinischen Kuchen erstarrt. Ich habe mit Rücksicht auf die geringe Menge der Substanz und dem constanten Siedepunkt eine weitere Reinigung nicht vorgenommen. Der Körper schmilzt bei $49^{\circ}$ (uncorr.). 
Die Analyse der Verbindung ergab die folgenden Zahlen:

1. $0.26 g$ Substanz lieferten $0.5284 \mathrm{~g}$ Kohlensäure und $0.1195 \mathrm{~g}$ Wasser.

II. $0.2513 \mathrm{~g}$ Substanz lieferten $0.2096 \mathrm{~g}$ Jodsilber nach $Z$ e is e 1 .

III. $0.2103 \mathrm{~g}$ Substanz lieferten $0 \cdot 1344 \mathrm{~g}$ Essigsäure nach $\mathrm{W}$ en $z$ el.

Auf 100 Theile:

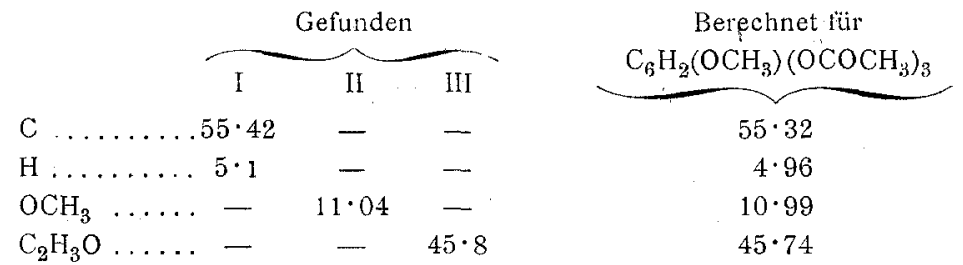

Die Acetylirung des Iretols führt somit zur Bildung eines Triacetylderivates.

Die guten Erfolge, von denen der Versuch, das Iretol synthetisch zu gewinnen, begleitet war, haben mich veranlasst, auch die Darstellung des entsprechenden Phentetroläthyläthers vorzunehmen. Das Ausgangsproduct für dessen Gewinnung, den Pikrinsäureäthyläther, habe ich nach den Angaben von Aussen ${ }^{1}$ aus reinem Pikrylchlorid in absolut alkoholischer Lösung mit Natrium gewonnen. Das Product wurde wiederholt aus Alkohol umkrystallisiri und zeigte den richtigen Schmelzpunkt $\left(78 \cdot 5^{\circ}\right)$.

\section{Reduction des Pikrinsäureäthyläthers.}

Was die Details der Reaction betrifft, so verweise ich auf das beim Methyläther Gesagte. Das erhaltene Product gleicht in seinen Eigenschaften durchaus dem entsprechenden Methylderivat; gleich diesem färbt es sich an der Luft blassviolett und liefert mit verdünnter Eisenchloridlösung und mit verdünnter Ammoniaklösung keine charakteristische Färbung. Dagegen liefert das bei Anwendung von concentrirter Salzsäure und unter starker Erwärmung (vergl. S. 928) gewonnene Product mit Eisenchlorid auch bei starker Verdünnung eine blaue odet blauviolette Färbung. Es liegt nahe, zu vermuthen, dass diese

1 Berl. Ber., 8, 666. 
Reaction von geringen Mengen von Triamidophenol herrührt, welches bei der absoluten Reinheit des angewendeten Ausgangsmateriales sich nur durch eine Nebenreaction gebildet haben könnte. Mit verdünnter Ammoniaklösung entsteht bei demselben Producte eine sehr intensiv blaue Färbung; dieselbe Farbenreaction gibt auch das von Nietzki und Moll (Berl. Ber., 26, 2184) dargestellte $(1,2,4,6)$-Diamidobrenzkatechin; es wäre daher möglich, dass auch dieser Körper durch Nebenreaction sich bildet, sei es durch Hydrolysirung des Triamidophenols, sei es durch Abspaltung von Chloräthyl aus dem Diamidodioxybenzoläthyläther. Oben erwähnte Farbenreaction zeigt übrigens das unter den gleichen Bedingungen gewonnene Reductionsproduct des Pikrinsäuremethyläthers.

Eine sichere Deutung dieser Erscheinung und zugleich die Constitutionsermittlung des Amidoproductes wird erst möglich sein durch eingehendes Studium der Producte, welche durch Abspaltung von Chlormethyl, beziehungsweise Chloräthyl aus den Diamidodioxybenzoläthern sich bilden; ich muss die Erledigung dieser Frage in Folge Mangels an Zeit und Material auf einen späteren Zeitpunkt verschieben.

I. $0 \cdot 2287 \mathrm{~g}$ Substanz gaben $0 \cdot 3064 \mathrm{~g}$ Kohlensäure und $0 \cdot 139 \mathrm{~g}$ Wasser.

II. $0: 2405 \mathrm{~g}$ Substanz gaben $0.3268 \mathrm{~g}$ Kohlensäure und $0.1293 \mathrm{~g}$ Wasser.

III. $0.2319 \mathrm{~g}$ Substanz gaben $20.8 \mathrm{~cm}^{3}$ feuchten Stickstoff bei $15^{\circ} \mathrm{C}$. und $751 \cdot 1 \mathrm{~mm}$ Druck.

IV. $0.225 g$ Substanz gaben $21 \mathrm{~cm}^{3}$ feuchten Stickstoff bei $18^{\circ} \mathrm{C}$. und $753 \cdot 8 \mathrm{~mm}$ Druck.

V. $0.2093 \mathrm{~g}$ Substanz gaben $0 \cdot 2360 \mathrm{~g}$ Chlorsilber.

VI. $0.2353 \mathrm{~g}$ Substanz gaben $0 \cdot 260 \mathrm{~g}$ Chlorsilber.

VII. $0 \cdot 2305 \mathrm{~g}$ Substanz gaben $0 \cdot 2096 \mathrm{~g}$ Jodsilber nach $Z$ e ise 1 .

Auf 100 Theile:

\begin{tabular}{|c|c|c|c|c|c|c|}
\hline I & II & III & IV & $\mathrm{V}$ & VI & Vll \\
\hline$\ldots \ldots .36 \cdot 54$ & $37 \cdot 06$ & - & - & - & - & - \\
\hline$\ldots \ldots 6.07$ & $5 \cdot 97$ & - & - & - & - & - \\
\hline$\cdots-$ & - & $10 \cdot 37$ & $10 \cdot 69$ & - & - & - \\
\hline$\ldots \ldots \ldots-$ & - & - & - & $27 \cdot 88$ & $27 \cdot 28$ & - \\
\hline $\mathrm{OC}_{2} \mathrm{H}_{5} \quad \ldots \quad-$ & - & - & - & -- & - & $17 \cdot 471$ \\
\hline
\end{tabular}

1 Die Analysen I, III und $V$ sind mit einer Substanz von einer Darstellung $A$, II, IV, VI, VII von einer Darstellung $B$ ausgeführt worden. 
Wie beifolgende Tabelle zeigt, stimmen die einander entsprechenden Zahlen nicht alle genau untereinander überein and sind von den für das zu erwartende. Chlorhydrat des Dioxydiamidobenzoläthyläthers berechneten wesentlich abweichend; dagegen stehen sie zum grossen Theile mit: den Zahlen in Übereinstimmung, die sich bei der Annahme eines Moleküls Krystallwasser ergeben.

Gefunden

$$
\text { A } \overbrace{B}
$$

C $\ldots \ldots \ldots 36: 54 \quad 37 \cdot 06$

$\mathrm{HI} \ldots \ldots \ldots 6 \cdot 07 \quad 5 \cdot 97$

N . . . . . 10.37 $\quad 10 \cdot 69$

Cl......27 $2788 \quad 27 \cdot 28$

$\mathrm{OC}_{2} \mathrm{H}_{5} \ldots \ldots-17 \cdot 47$
Berechnet für

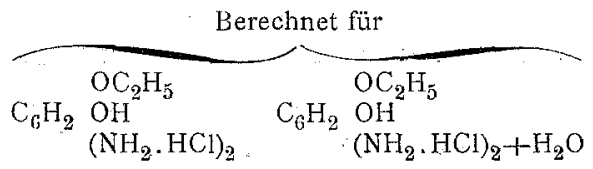

$39 \cdot 87$

$5 \cdot 81$

$11 \cdot 63$

$29 \cdot 4$

$18 \cdot 68$
$37 \cdot 05$

$6 \cdot 18$

$10 \cdot 43$

$27 \cdot 35$

$17 \cdot 38$

Ich habe zur Gontrole die Krystallwasserbestimmung ausgeführt, indem ich die Substanz (Darstellung $B$ ) im partiellen Wasserstoffvacuum auf $100^{\circ}$ bis zur Gewichtsconstanz erhitzte.

$0.3189 \mathrm{~g}$ : Substanz gaben $0.0246 \mathrm{~g}$ Wasser $=7 \cdot 71 \%$, während obige Formel $7 \cdot 4 \%$ erfordert.

Die Verbrennung der auf diese Weise getrockneten Substanz ergab folgende Werthe:

$0.2908 g$ Substanz lieferten $0.4273 g$ Kohlensäure und $0.1518 g$ Wasser.

Auf 100 Theile:

Berechnet für
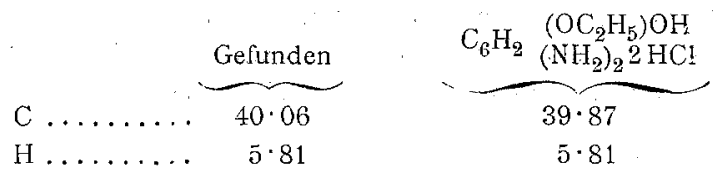

Durch diese Zahlen ist das Vorhandensein eines Moleküls Krystallwasser erwiesen.

\section{Einwirkung von Wasser auf das Chlorhydrat des Diamido- dioxybenzoläthyläthers.}

Auch hier verläuft die Reaction ganz analog wie beim Methyläther. Ich habe nur zu bemerken dass man den Tetraoxybenzoläthyläther aus dem Rohproduct nach partieller Fällung. 
mit Bleiacetat am besten in der Weise gewinnt, dass man die vom Schwefelblei abfiltrirte, fast farblose Flüssigkeit nahezu bis zur Trockene eindampft und den Körper, welcher sich in schwach gelb gefärbten Krystallkrusten ausscheidet, mit wenig kaltem Wasser aus dem Kolben spült. Das Wasser nimmt dabei die gefärbten Verunreinigungen auf und man erhält nach dem Absaugen die Verbindung in farblosen Krystallnadeln vom Schmelzpunkte $220^{\circ}$ (uncorr.).

In seinen Löslichkeitsverhältnissen gleicht der Körper dem Iretol; ebenso wie dieses gibt er eine Fichtenspahn- und Eisenchloridreaction.

Die Analyse der bei $100^{\circ}$ getrockneten Substanz ergab die folgenden Werthe:

I. $0.2374 \mathrm{~g}$ Snbstanz gaben $0.4883 \mathrm{~g}$ Kohlensäure und $0.1207 \mathrm{~g}$ Wasser. II. $0.2120 \mathrm{~g}$ Substanz gaben $0.2911 \mathrm{~g}$ Jodsilber nach $Z$ eisel.

Auf 100 Theile:

\begin{tabular}{|c|c|c|}
\hline$\frac{\text { Gefi }}{\mathrm{I}}$ & $\frac{\text { nden }}{\text { II }}$ & $\begin{array}{c}\text { Berechnet für } \\
\mathrm{C}_{6} \mathrm{H}_{2}\left(\mathrm{OC}_{2} \mathrm{H}_{5}\right)(\mathrm{OH})_{3}\end{array}$ \\
\hline $\mathrm{C} \ldots \ldots \ldots 56 \cdot 11$ & - & $56 \cdot 47$ \\
\hline H $\ldots \ldots \ldots 5.63$ & - & $5 \cdot 88$ \\
\hline $\mathrm{OC}_{2} \mathrm{H}_{5} \ldots \ldots-$ & $26 \cdot 26$ & $26 \cdot 47$ \\
\hline
\end{tabular}

Aus der Übereinstimmung der gefundenen mit den berechneten Zahlèn ergibt sich, dass die Hydrolyse des Diamidodioxybenzoläthyläthers $z u$ dem erwarteten Tetraoxybenzoläthyläther führt.

Da mir nur eine sehr geringe Menge Substanz zur Verfügung stand, musste ich mich auf die Darstellung des Acetylproductes beschränken.

\section{Einwirkung von Essigsäureanhydrid auf den Tetraoxybenzol- äthyläther.}

Unter denselben Bedingungen wie beim Iretol erhielt ich ein Acetylproduct, welches durch Destillation im Vacuum gereinigt wurde. Dasselbe ging bei $232^{\circ}$ unter $17 \mathrm{~mm}$ über und erstarrte nach kurzer Zeit krystallinisch. Die Substanz wurde in Benzol gelöst und mit Ligroin bis zur beginnenden 
Trübung versetzt; es schieden sich alsbald farblose Krystalle aus, welche abgesaugt und getrocknet, den Schmelzpunkt $74^{\circ}$ zeigten.

Die Analyse der im Vacuum über Öl getrockneten Substanz ergab die folgenden Werthe:

I. $0.251 \mathrm{~g}$ Substanz gaben $0.5273 \mathrm{~g}$ Kohlensäure und $0.1159 \mathrm{~g}$ Wasser.

II. $0 \cdot 218 g$ Substanz gaben $0 \cdot 1714 g$ Jodsilber nach $Z$ e is el.

Auf 100 'Theile:
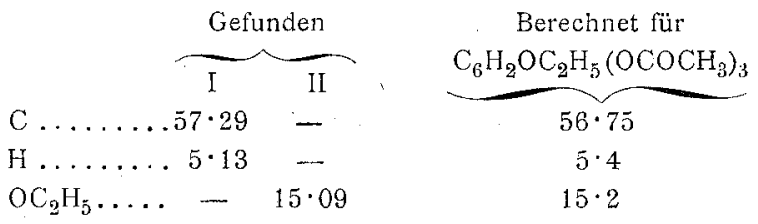

Die Acetylirung des 1, 2, 4, 6-Phentetroläthyläthers führt mithin zur Bildung eines Triacetylderivates.

Der Vergleich der Schmelzpunkte der correspondirenden Äther der Nitrokörper, Phenole und Phenacylderivate zeigt eine ziemlich ausgesprochene Gesetzmässigkeit.

\begin{tabular}{|c|c|c|}
\hline & Methyläther & Äthyläther \\
\hline Pikrinsäure . . . . . . & $66^{\circ}$ & $78 \cdot 5^{\circ}$ \\
\hline Phentetrol . . . . . . . . & 186 & 220 \\
\hline Triacetylphentetro1...... & 49 & 74 \\
\hline
\end{tabular}

Das Äthoxylderivat schmilzt durchgehends höher als das Methoxylderivat, und die Schmelzpunktsdifferenz wird am grössten bei den Phenolen $\left(34^{\circ}\right)$; die grösste Schmelzpunkterhöhung hat der Ersatz der Nitrogruppen durch Hydroxyle zur Folge. Durch Eintritt von drei Acetylgruppen wird der Schmelzpunkt wieder unter den der entsprechenden Nitroverbindungen herabgedrückt.

Die Resultate der vorliegenden Arbeit rechtfertigen die von mir ausgesprochene Erwartung, das Triamidophenol werde 
sich nach Ätherificirung der Hydroxylgruppe ähnlich einem Homologen des Triamidobenzols verhalten.

Ebenso wie die Methylgruppe des Triamidotoluols sich bei der Einwirkung von Wasser ziemlich passiv verhält, so dass ein wesentlicher Unterschied im Verhalten desselben vom Triamidobenzol nicht beobachtet werden kann, zeigt sich im Reagiren der Reductionsproducte der Pikrinsäureäther gegen Wasser ein viel geringerer Unterschied im Vergleich zum Triamidotoluol als im Vergleich zum Triamidophenol selbst; ja die Tendenz zur Hydrolysirung ist so gross, dass schon bei der Reduction eine Amidogruppe durch Hydroxyl ersetzt wird. Wenn gleichwohl die Ausbeuten, in denen die Phentetroläther erhalten wurden, $25 \%$ nicht überschritten, so kann dies nut dem Umstande zugeschrieben werden, dass diese Körper namentlich in unreinem Zustande durch Wasser leicht zersetzt werden, worauf schon de Laire und Tiemann aufmerksam machen.

Die Thatsache aber, dass der Bestand der Methoxylgruppe und Äthoxylgruppe durch den Reductionsprocess und die langdauernde Einwirkung von Wasser nicht gefährdet wird, berechtigt zur Hoffnung, dass es gelingen wird, auch aus den Äthern des Trinitroresorcins und Trinitrophloroglucins $z u$ den entsprechenden Phenoläthern zu gelangen. Die Herstellung dieser Verbindungen wird der Gegenstand weiterer Untersuchungen sein.

Vorliegende Arbeit ist auf Anregung und unter Leitung meines nunmehr verewigten Lehrers Prof. $\mathrm{H}$. Weidel gemacht worden. Mit Wehmuth gedenke ich dankbar der Liebenswürdigkeit, mit der er mir bei der Ausführung der beschriebenen Versuche stets beizustehen die Güte hatte. 